\title{
INDUCTION AND STRUCTURE THEOREMS FOR GROTHENDIECK AND WITT RINGS OF ORTHOGONAL REPRESENTATIONS OF FINITE GROUPS
}

\author{
BY ANDREAS DRESS
}

Communicated by Hyman Bass, November 17, 1972

\begin{abstract}
The Grothendieck- and Witt- ring of orthogonal representations of a finite group is defined and studied. The main application (only indicated) is the reduction of the computation of Wall's various $L$-groups for a finite group $\pi$ to those subgroups of $\pi$, which are a semidirect product of a cyclic group $\gamma$ of odd order with a 2 -group $\beta$, such that any element in $\beta$ acts on $\gamma$ either by the identity or by taking any element in to its inverse.
\end{abstract}

Let $\pi$ be a finite group and $R$ a Dedekind ring. An $R \pi$-lattice $(M, f)$ or just $M$ is defined to be a finitely generated, $R$-projective $R \pi$-module $M$ together with a symmetric, $\pi$-invariant nonsingular form $f: M \times M \rightarrow R$ (cf. [3]). For two $R \pi$-lattices $M_{1}$ and $M_{2}$ one has their orthogonal sum $M_{1} \perp M_{2}$ and tensor product $M_{1} \otimes M_{2}$, thus the isomorphism classes of $R \pi$-lattices form a half-ring $Y^{+}(R, \pi)$, whose associated Grothendieck ring is denoted by $Y(R, \pi)$. For a subgroup $\gamma \leqq \pi$ one has in an obvious way, restriction and induction of $R \pi$-lattices, resp. $R \gamma$-lattices, and it is easily seen (cf. [3]) that this makes $Y(R,-)$ into a $G$-functor in the sense of Green (cf. [5]).

As in the theory of integral group-representations, where the Grothendieck ring of isomorphism classes of $R \pi$-modules is much too large for many purposes and is thus replaced by its quotient $G_{0}(R, \pi)$ (in the sense of [9]) modulo the ideal, generated by the Euler characteristics of short exact sequences of $R \pi$-modules, we are going to define certain quotients of $Y(R, \pi)$, using a relation which was first introduced by $\mathrm{D}$. Quillen in $[7, \S 5]$. At first let us remark, that for any finitely generated $R$ projective $R \pi$-module $N$, one has the associated hyperbolic module $H(N)=\left(N \oplus N^{*}, f\right)$ with $N^{*}=\operatorname{Hom}_{R}(N, R)$ the $R$-dual of $N$, considered as $R \pi$-module (with $(g \cdot v)(n)=v\left(g^{-1} \cdot n\right), g \in \pi, v \in N^{*}, n \in N$ ).and $f(N, N)=f\left(N^{*}, N^{*}\right)=0, f(n, v)=v(n), n \in N, v \in N^{*}$. We now define a Quillen pair $(M, N)$ to be an $R \pi$-lattice $M=(M, f)$ together with an

AMS (MOS) subject classifications (1970). Primary 15A63, 16A54, 18F25; Secondary 20C99, 20J99, 57D65.

Key words and phrases. Orthogonal representations of finite groups, $L$-groups, Witt ring, induced representations, Frobenius-functor, quadratic forms. 
$R \pi$-submodule $N \subseteq M$, such that $N$ is an $R$-direct summand (i.e., $M / N$ and thus also $N$ is $R$-projective) and $f(N, N)=0$. For example, $(H(N), N)$ and $\left(H(N), N^{*}\right)$ are Quillen pairs. If $(M, N)$ is a Quillen pair, then $N \subseteq N^{\perp}=\{m \in M \mid f(m, N)=0\}$ and $N^{\perp} / N$ is an $R \pi$-lattice again.

Let $I_{1}$, resp. $I_{2}, \subseteq Y(R, \pi)$ be the ideal, generated by all elements of the form $[M]-\left[N^{\perp} / N\right]-[H(N)]$, resp. $[M]-\left[N^{\perp} / N\right]$, where $(M, N)$ is a Quillen pair, and define $G U_{0}(R, \pi)=Y(R, \pi) / I_{1}, G W_{0}(R, \pi)=Y(R, \pi) / I_{2}$. Because $H(N)=0$ in $G W_{0}(R, \pi)$ one has $I_{1} \subseteq I_{2}$ and thus a natural surjection $G U_{0}(R, \pi) \rightarrow G W_{0}(R, \pi)$. Moreover one checks easily, that the hyperbolic construction defines a well-defined map

$$
H: G_{0}(R, \pi) \rightarrow G U_{0}(R, \pi),
$$

whose image is precisely the kernel of $G U_{0}(R, \pi) \rightarrow G W_{0}(R, \pi)$, i.e., we have a natural exact sequence $G_{0}(R, \pi) \rightarrow G U_{0}(R, \pi) \rightarrow G W_{0}(R, \pi) \rightarrow 0$.

Finally the $G$-functor structure on $Y(R,-)$ carries through to a $G$ functor structure on $G U_{0}(R,-)$ and on $G W_{0}(R,-)$ and the above sequence behaves well with respect to restriction and induction.

Now for a set $\mathfrak{H}$ of subgroups of $\pi$ define $I\left(\mathfrak{H}, G W_{0}\right)$, resp. $I\left(\mathfrak{H}, G U_{0}\right)$, to be the sum of the images of $G W_{0}(R, \gamma)$, resp. $G U_{0}(R, \gamma),(\gamma \in \mathfrak{G})$ in $G W_{0}(R, \pi)$, resp. $G U_{0}(R, \pi)$, with respect to the induction maps from $\gamma$ to $\pi$. For a set $\Sigma$ of prime numbers let $\mathfrak{S}_{\Sigma}(\pi)$ be the set of subgroups of $\pi$, which are $p$-hyperelementary (i.e. have a cyclic normal subgroup of $p$-power index) for some $p \in \Sigma$, especially $\mathfrak{H}_{\phi}(\pi)=\{\gamma \leqq \pi \mid \gamma$ cyclic $\}$. For the order $|\pi|=\prod p^{\alpha p}$ of $\pi$ define $|\pi|_{\Sigma}=\prod_{p \in \Sigma} p^{\alpha p},|\pi|_{\Sigma^{\prime}}=\prod_{p \notin \Sigma} p^{\alpha p}$ (thus $\left.|\pi|=|\pi|_{\Sigma} \cdot|\pi|_{\Sigma^{\prime}}\right)$. Then we have

THEOREM 1. $n \cdot 1_{G W_{0}(R, \pi)} \in I\left(\mathfrak{H}_{\Sigma}(\pi), G W_{0}\right)$ with $n=|\pi|_{\Sigma^{\prime}}$ for $|\pi|_{\Sigma^{\prime}}$ odd and $n=4 \cdot|\pi|_{\Sigma^{\prime}}$ in any case.

THEOREM 2. $n \cdot 1_{G U_{0}(R, \pi)} \in I\left(\mathfrak{H}_{\Sigma}(\pi), G U_{0}\right)$ with

$$
\begin{aligned}
n & =|\pi|_{\Sigma^{\prime}}^{2} & & \text { for } R \text { semilocal, }|\pi|_{\Sigma^{\prime}} \text { odd }, \\
& =4 \cdot|\pi|_{\Sigma^{\prime}}^{2} & & \text { for } R \text { semilocal }, \\
& =|\pi|_{\Sigma^{\prime}}^{3} & & \text { for }|\pi|_{\Sigma^{\prime}} \text { odd }, \\
& =4 \cdot|\pi|_{\Sigma^{\prime}}^{3} & & \text { in any case. }
\end{aligned}
$$

Since $G U_{0}(R, \pi)$ acts naturally as a Frobenius-functor on most (if not all) of the various $L$-groups, associated with a finite group $\pi$ (cf. [11]), one thus can reduce the study of these $L$-groups to the case of $p$-hyperelementary groups. Actually for any such $L$-functor-let it be called just $L-$ one has

COROLLARY 1. The various restriction maps define an isomorphism of 
$L_{\Sigma}(\pi)=_{\mathrm{Df}} Z[1 / p \mid p \notin \Sigma] \otimes L(\pi)$ onto the subgroup of $\prod_{\gamma \in \mathfrak{S}_{\Sigma}(\pi)} L_{\Sigma}(\gamma)$, consisting of those tuples $\left(x_{\gamma}\right)_{\gamma \in \mathfrak{S}_{\Sigma}(\pi)}$ with $x_{\gamma} \in L_{\Sigma}(\gamma), x_{\gamma \mid \delta}=x_{\delta}$ for any $\delta \leqq \gamma$ and $x_{\gamma}^{g}=x_{g_{\gamma g}{ }^{-1}}$ for any $g \in \pi\left(x_{\gamma \mid \delta}\right.$ the restriction from $\gamma$ to $\delta, x \rightarrow x^{g}$ the natural isomorphism from $L_{\Sigma}(\gamma)$ onto $L_{\Sigma}\left(g \gamma g^{-1}\right)$, associated with $\left.g\right)$.

Proof. This follows the same way from Theorem 2 as R. Brauer's characterization of generalized characters among class functions by their restriction to elementary subgroups from his induction theorem. Thus it can also be considered as a special case of $[1, \S 8$, Appendix $]$.

Using the above exact sequence and Swan's induction theorems for $G_{0}(R, \pi)$ (cf. [10]), Theorem 2 follows by a well-known trick, due to Swan (cf. the proof of Proposition 1 in [10, pp. 558-559]), from Theorem 1. Theorem 1 itself follows to some extent from the following result on the structure of $G W_{0}(R, \pi)$ :

THEOREM 3. For any ring $A$ write $A^{\prime}$ for $Z\left[\frac{1}{2}\right] \otimes A$. Then

(i) $G W_{0}(\pi)^{\prime}={ }_{\mathrm{Df}} G W_{0}(\boldsymbol{Z}, \pi)^{\prime} \cong G_{0}(\boldsymbol{R}, \pi)^{\prime}$.

(ii) $G W_{0}(R, \pi)^{\prime} \cong G W_{0}(\pi)^{\prime} \otimes_{\mathbf{Z}} W(R)^{\prime}$ (with $W(R)=G W_{0}(R, \varepsilon)-\varepsilon$ the trivial group - the Witt ring of $R$ in the sense of Knebusch [6]).

(iii) The torsion subgroup of $G W_{0}(\pi)$ is annihilated by 4 .

Indeed, using the general theory of the Burnside ring (cf. [1, especially $\S 8$, Theorem 8.2]), Theorem 3 implies Theorem 1 with $n=4 \cdot|\pi|_{\Sigma^{\prime}}$. Theorem 3 itself follows from results of A. Fröhlich (cf. [4]) on $G W_{0}(R, \pi)$ for $R$ a field of characteristic 0 and from

Proposition 1. If $K$ is the quotient field of $R$, then the natural map $G W_{0}(R, \pi) \rightarrow G W_{0}(K, \pi)$ is injective. Furthermore, if $R$ has no formally real residue class field, then $G W_{0}(R, \pi)^{\prime} \rightarrow G W_{0}(K, \pi)^{\prime}$ is an isomorphism.

PROOF. Straightforward generalization of the argument for Satz 11.1.1 in [6]. Another way to prove Theorem 3 is to combine Proposition 1 with

Proposition 2. Let $L$ be a finite Galois extension of a field $K$ with Galois group $\mathfrak{6}$, such that any ordering of $K$ can be extended to $L$. Then we have $G W_{0}(K, \pi)^{\prime} \cong\left(G W_{0}(L, \pi)^{\prime}\right)^{\mathfrak{G}}$ for the natural action of $\left(\mathfrak{5}\right.$ on $G W_{0}(L, \pi)^{\prime}$.

This follows from [1, Appendix B, Theorem 3.2], using Scharlau's induction technique for Witt rings (cf. [8] and also [1, Appendix A]).

PROPOSITION 3. Let us call a formally real field $K$ a real splitting field for the group $\pi$, if for any irreducible $K \pi$-module $N$ and any formally real extension $L$ of $K$ the module $L \otimes_{K} N$ is an irreducible $L \pi$-module. Then

(i) $G_{0}(K, \pi) \cong G_{0}(R, \pi)$,

(ii) $G W_{0}(K, \pi)^{\prime} \cong G_{0}(K, \pi)^{\prime} \otimes_{z} W(K)^{\prime}$.

Moreover if $n=\exp (\pi), \xi$ a primitive nth root of unity and $K$ any formally 
real field, then $K\left(\xi+\xi^{-1}\right)$ is a real splitting field of $\pi$.

Propositions 1, 2 and 3 now imply the most important part of Theorem 3

$$
\begin{aligned}
G W_{0}(\pi)^{\prime} & =G W_{0}(\boldsymbol{Z}, \pi)^{\prime} \\
& \cong G W_{0}(\boldsymbol{Q}, \pi)^{\prime} \cong\left(G W_{0}\left(\boldsymbol{Q}\left(\xi+\xi^{-1}\right), \pi\right)^{\prime}\right)^{\mathfrak{5}} \\
& \cong\left(G_{0}\left(\boldsymbol{Q}\left(\xi+\xi^{-1}\right), \pi\right)^{\prime} \otimes_{Z} W\left(\boldsymbol{Q}\left(\xi+\xi^{-1}\right)\right)^{\prime}\right)^{\mathfrak{G}} \\
& \cong\left(G_{0}(\boldsymbol{R}, \pi)^{\prime} \otimes_{Z} W\left(\boldsymbol{Q}\left(\xi+\xi^{-1}\right)\right)^{\prime}\right)^{\mathfrak{G}} \\
& \cong G_{0}(\boldsymbol{R}, \pi)^{\prime} \otimes_{Z}\left(W\left(\boldsymbol{Q}\left(\xi+\xi^{-1}\right)\right)^{\prime}\right)^{\mathfrak{5}} \\
& \cong G_{0}(\boldsymbol{R}, \pi)^{\prime} \otimes_{Z} W(\boldsymbol{Q})^{\prime} \cong G_{0}(\boldsymbol{R}, \pi)^{\prime},
\end{aligned}
$$

especially all torsion in $G W_{0}(\pi)$ is 2-torsion. The other parts of Theorem 3 need some more care. (But for $R$ a field $K$ one has of course

$$
\begin{aligned}
W G_{0}(K, \pi)^{\prime} & \cong\left(W G_{0}\left(K\left(\xi+\xi^{-1}\right), \pi\right)^{\prime}\right)^{\mathfrak{5}} \\
& \cong\left(G_{0}\left(K\left(\xi+\xi^{-1}\right), \pi\right)^{\prime} \otimes W\left(K\left(\xi+\xi^{-1}\right)\right)^{\prime}\right)^{\mathfrak{5}} \\
& \cong\left(G_{0}(\boldsymbol{R}, \pi)^{\prime} \otimes W\left(K\left(\xi+\xi^{-1}\right)\right)^{\prime}\right)^{\mathfrak{5}} \\
& \cong\left(G W_{0}(\pi)^{\prime} \otimes W\left(K\left(\xi+\xi^{-1}\right)\right)^{\prime}\right)^{\mathfrak{G}} \\
& \cong G W_{0}(\pi)^{\prime} \otimes\left(W\left(K\left(\xi+\xi^{-1}\right)\right)^{\prime}\right)^{\mathfrak{G}} \cong G W_{0}(\pi)^{\prime} \otimes W(K)^{\prime} ;
\end{aligned}
$$

thus the same holds as well for Dedekind rings with no formally real residue class field. It also shows that for any field $K$ all torsion in $G W_{0}(K, \pi)$ is 2-torsion, which was conjectured by A. Fröhlich in [4].)

To get rid of the factor 4 in Theorem 1 for $|\pi|_{\Sigma}$, odd, one has to use multiplicative induction theory as developed, for instance, in [2]. Reducing trivially to the case $\Sigma=\{2\}$ and using this technique, it is enough to prove the corresponding statement for groups of rather simple types: elementary abelian groups of order $p^{2}$ ( $p$ odd), nonabelian groups of order $p \cdot q$ ( $p, q$ odd primes) and semidirect products of cyclic groups of order $p$ with elementary abelian 2-groups, on which the cyclic group of order $p$ acts nontrivially and irreducibly. But in all these cases the torsion part of $G W_{0}(\pi)$ is easily shown to be nilpotent and thus one can use the fact that, in case all torsion elements in $G W_{0}(R, \pi)$ are nilpotent, the wanted result follows directly from Theorem 3 by AGN-methods and Burnside ring theory (cf. [1, especially $\S 8$, Theorem 8.2]). Actually I conjecture that for any group $\pi$ all torsion elements in $G W_{0}(R, \pi)$ are nilpotent. This would allow us to avoid multiplicative induction techniques in this case completely; on the other hand, our induction theorem reduces this question to the case of 2-hyperelementary groups. I can prove the conjecture for a great number of special classes of groups, but right now 
it seems to me, that a proof in the general case might be as complicated and even more involved than the multiplicative induction techniques I am using now.

\section{REFERENCES}

1. A. Dress, Notes on the theory of representations of finite groups. I: The Burnside ring of a finite group and some AGN-applications, Multicopied lecture notes, Bielefeld, 1971.

2. - Contributions to the theory of induced representations, Proc. Conf. Algebra K-theory, (Seattle, Wash., 1972), Lecture Notes in Math., Springer-Verlag (to appear).

3. A. Fröhlich and A. M. McEvett, The representations of groups by automorphisms of forms, J. Algebra 12 (1969), 114-133.

4. A. Fröhlich, Orthogonal and symplectic representations of groups, Proc. London Math. Soc. (3) 24 (1972), 470-506.

5. I. A. Green, Axiomatic representation theory for finite groups, J. Pure Appl. Algebra 1 (1971), 41-77.

6. M. Knebusch, Grothendieck- und Wittringe von nicht ausgearteten symmetrischen Bilinearformen, S. -B. Heidelberger Akad. Wiss. Math.-Natur. K 1969/70, 93-157. MR 42 \#6001.

7. D. G. Quillen, The Adams conjecture, Topology 10 (1971), 67-80.

8. W. Scharlau, Induction theorems and the structure of the Witt group, Invent. Math. 11 (1970), 37-44.

9. R. Swan, K-theory of finite groups and orders, Lecture Notes in Math., vol. 149, Springer-Verlag, Berlin and New York, 1970.

10. Induced representations and projective modules, Ann. of Math. (2) 71 (1960), 552-578. MR 25 \#2131.

11. C. T. C. Wall, Foundations of algebraic L-theory, Liverpool, 1972 (preprint).

Department of Mathematics, University of Bielefeld, Bielefeld, Federal RePUBLIC OF GERMANY 